\title{
Clinical course of ectopic pregnancy in a tertiary center: Observational study
}

\author{
Pratiksha Gupta ${ }^{1}$, Sanjana Narula Wadhwa ${ }^{2 *}$ \\ ${ }^{1}$ Professor, ${ }^{2}$ Assistant Professor, ${ }^{1,2}$ Dept. of Obstetrics and Gynecology, ${ }^{1,2}$ ESIC, PGIMSR Basaidarapur, Delhi, India
}

Corresponding author: Sanjana Narula Wadhwa

Email: dr.sanjana297@gmail.com

Received: $25^{\text {th }}$ December, 2018

Accepted: $8^{\text {th }}$ May, 2019

\begin{abstract}
Objective: Ectopic pregnancy is a life-threatening obstetric emergency. The objective of the study was to highlight the incidence, clinical profile and the management of ectopic pregnancy.

The study was conducted in Obstetrics and Gynecology department of ESIC PGIMSR Delhi over one year. It was an observational study.

Materials and Methods: Pregnant women presenting in gynaecology emergency with pain abdomen or bleeding per vaginum or history suggestive of ectopic pregnancy in first trimester were assessed for intrauterine or extrauterine pregnancy. Written informed consent was obtained from each patient prior to the treatment. All pregnant women with intrauterine pregnancies were excluded. 77 pregnant women were found to have extrauterine pregnancy. All Hemodynamically stable cases were managed conservatively. Hemodynamically compromised cases were managed surgically. Surgical procedure performed was salpingectomy. These cases were followed with weekly beta hCG till it normalized.

Outcome: Incidence, Age, Parity and the treatment given.

Results: Incidence of ectopic pregnancy in the institution was $2.01 \% .46 .7 \%$ cases were among age group 20-30 years. Multigravida $46.7 \%$ were having more ectopic pregnancy. $99 \%$ of extrauterine pregnancy were located in the Fallopian tubes. 38.9\% presented between $4-6$ weeks period of gestation $10.3 \%$ managed conservatively. $11.6 \%$ were chronic ectopic pregnancies were operated. Salpingectomy was the main surgical procedure.

Conclusion: Thus, high index of clinical suspicion of ectopic pregnancy must be kept in mind. Early diagnosis and prompt treatment can help in reducing maternal mortality and morbidity.
\end{abstract}

Keywords: Ectopic pregnancy, Salpingectomy, Fallopian tube.

\section{Introduction}

Ectopic pregnancy is an important cause of maternal morbidity and mortality in India. It is defined as implantation of fertilized egg that is embryo, outside the uterine endometrium, and fetus and placenta develop there.

The incidence of the ectopic pregnancy is around 1-2\% of all the pregnancies. Incidence of extrauterine pregnancy increases to $4 \%$ if pregnancy involves assisted reproductive technology. ${ }^{1}$

Between 6 to 16 percent of pregnant women who visit emergency department in the first trimester for bleeding, pain or both, have ectopic pregnancy. In developing nations, woman usually presents late with hemodynamic instability.

Any woman in her reproductive age group, presenting with pain lower abdomen or bleeding per vaginum, with or without history of amenorrhea, must raise the suspicion ectopic pregnancy to prevent mortality aand mortality.

Through this study we aimed to find the incidence of ectopic pregnancy and how the early diagnosis of the ectopic pregnancy can prevent morbidity and mortality in a tertiary centre.

\section{Material and Methods}

This was a prospective study which was undertaken at the Department of Obstetrics and Gynaecology of ESIC PGIMSR Basaidarapur, Delhi. Study was conducted over one-year January 2016 to March 2017. All pregnant women presenting in gynaecology emergency department with pain abdomen or bleeding per vaginum or history suggestive of ectopic pregnancy in first trimester were assessed for intrauterine or extrauterine pregnancy. All blood investigations and ultrasound pelvis were done. Written informed consent was obtained from each patient prior to the treatment. All pregnant women with intrauterine pregnancies were excluded from the study. 77 pregnant women were found to have extrauterine pregnancy. All cases had serum beta hCG levels measured at the time of admission as baseline along with other blood investigations. These cases were managed according to the hemodynamic status at the time of presentation to emergency department. Hemodynamically stable cases were managed conservatively. All the cases had the pelvic ultrasonography done to confirm the ectopic pregnancy. Those who fulfilled the medical management criteria that is: gestational size less than $4 \mathrm{~cm}$, normal haemoglobin with platelets and liver function test, beta hCG less than 5000iu/l were enrolled and taken for medical management. Medical management was done by administration of injection Methotrexate single dose. These were kept under follow up with weekly beta hCG till it normalized. Warning signs were explained regarding acute pain abdomen or fainting attacks to report to gynaecology department. Hemodynamically compromised cases were managed surgically. All the cases with rupture ectopic, hemodynamically instable, suspected heterotopic pregnancy were taken for surgical management. Surgical procedure performed was salpingectomy. These cases were followed with weekly beta hCG till it normalized. 
The outcomes were studied under the headings: Incidence, Age, Parity, Site of ectopic pregnancy, treatment given and number of blood products transfused.

\section{Results}

3830 pregnant women in their first trimester reported to the gynaecology emergency department during 15 months of study time. The chief complaint of these ladies were pain abdomen and amenorrhea associated with bleeding per vaginum or syncope which was suggestive of extrauterine pregnancy. After the detailed clinical history and physical examination and investigations, 77 cases were found to have ectopic pregnancy. Thus, the incidence of ectopic pregnancy in the present study was $2.01 \%$.

The $63.6 \%$ women were of age group 21 years to 30 years. $3.89 \%$ were above the 40 years age group.

$59.7 \%$ of the cases were multigravida. $46.7 \%$ presented between 4 weeks to 6 weeks of period of gestation.

$68.83 \%$ presented in emergency with the chief complaint of pain abdomen. The classical triad of symptoms of ectopic pregnancy that is pain abdomen, amenorrhea and bleeding per vaginum was observed in $12.98 \%$ cases.

On clinical examination, the abdominal tenderness $61.03 \%$ was the most prominent appreciable finding. $77.92 \%$ cases had ruptured ectopic pregnancy. $14.28 \%$ presented with amenorrhea and shock in the gynaecology emergency department.

$10.38 \%$ cases were hemodynamically stable and were managed conservatively. They all responded to the conservative management.

On the laparotomy, done for the ectopic pregnancy, intraoperatively ampullary region of the fallopian tube with the right sided was the site of ectopic pregnancy in $61.03 \%$ cases. Salpingectomy was the performed in $58.44 \%$ cases which were hemodynamically unstable and had ruptured ectopic pregnancy. $11.68 \%$ cases had chronic ectopic pregnancy.

$77.9 \%$ of cases received blood transfusion during or after the surgical procedure.

\section{Discussion}

The extrauterine pregnancy can be threatening condition to the maternal life.

The ectopic pregnancy is the implantation of fertilized ovum outside the uterus. The most common site for ectopic pregnancy is fallopian tubes $95 \%$. In the fallopian tubes, most commonly sites are in ampullary or isthmic portion of fallopian tubes. ${ }^{2}$ The nontubal extrauterine pregnancy and heterotopic pregnancy are rare (1-3\%). These tubal pregnancies are clinically important. ${ }^{3}$

The incidence of ectopic pregnancy worldwide is $0.25 \%$ to $2 \%{ }^{1}$ It is increasing with use of artificial reproduction techniques. In the present study, the incidence of ectopic pregnancy was $2.01 \%$.

The study conducted by Shukla DB et al in 2017 at a tertiary center found incidence of ectopic pregnancy was 1.209. ${ }^{4}$ Shanti et al (2016) found incidence of ectopic pregnancy $1.389 .{ }^{5}$ The incidence of ectopic pregnancy in the study of Porwal Sanjay et al was $2.46 .{ }^{6}$

The presentation of these extrauterine pregnancy is quite variable. These can be completely asymptomatic (intact tubal pregnancy [intact fallopian tube and embryo, perhaps with discernible cardiac function]), or it may present with pelvic pain that is worse on one side (tubal abortion) or with severe hemorrhagic shock (tubal rupture).

The classical triad of ectopic pregnancy characterized by pain, amenorrhea and vaginal bleeding is usually present in less than $50 \%$ of the cases.

In the present study, $12.98 \%$ had the classical triad. The triad was present in $21.5 \%$ cases in Shukla DB et al study, ${ }^{4}$ $50 \%$ cases had the classical triad in the study by Shanti et $\mathrm{al}^{5}$ and Rashmi et $\mathrm{al}^{8}$

Extrauterine pregnancies are commonly diagnosed in the 6th through 9th week of gestation; most patients present with nonspecific complaints ${ }^{9}$. In the present study $49.35 \%$ cases presented between 4-6 weeks of period of gestation. $31.16 \%$ cases were detected between $6-8$ weeks of period of gestation.

$62 \%$ were multiparous women presenting with ectopic pregnancy in study by Shanti $\mathrm{Et} \mathrm{al}^{5}$ and $62.2 \%$ were multiparous in Rashmi et $\mathrm{al}^{8}$ studies. $43.14 \%$ were nulliparous women had ectopic pregnancy in Shukla et al ${ }^{4}$.

In the present study, $77.97 \%$ cases were ruptured ectopic pregnancy and $77.97 \%$ received blood and blood product transfusions in the intraoperative period or postoperative period or both. In the study by Shanti et $\mathrm{al}^{5}$ and Porwal Sanjay et $\mathrm{al}^{6}$ all cases received blood products.

The ectopic pregnancy is major cause for the first trimester pregnancy related morbidity and mortality. It accounts for $6 \%$ pregnancy related mortality. ${ }^{9}$ Ectopic pregnancy accounts for $3.5-7.1 \%$ of maternal deaths in India. ${ }^{10,11}$

There was no maternal death due to ectopic pregnancy during the study period. Thus, the timely diagnosis and right intervention can prevent the fatalities caused by extrauterine pregnancy.

\section{Limitations}

Further study with a greater number of cases, to establish the role of conservative management and fertility sparing treatment in ectopic pregnancy is required.

\section{Conclusion}

Whenever a female in her reproductive age group presents with amenorrhea and pain abdomen with bleeding, High index of suspicion of extrauterine pregnancy should be kept in mind. All the high risk women for extrauterine pregnancy presenting with amenorrhea should be screened at the earliest with serum $\beta$-hCG and TVS. Timely diagnosis with active management helps to prevent the fatalities and complications in extrauterine pregnancy. The impact on future fertility can be improved by focusing on primary prevention and early diagnosis before rupture. 


\section{Acknowledgement}

Thankful to the patients and the Department of Obstetrics and Gynecology ESIC PGIMSR Delhi, for the cooperation in the study.

\section{Conflict of interests: None}

\section{Funding: None}

Ethics approval: Not required.

\section{Reference:}

1. Yadav A. Trends of ectopic pregnancies in Andaman and Nicobar Islands, Int J Reprod Contracept Obstet Gynecol 2017;6(1):15-19.

2. Howkins and Bourne Shaw's Text book of s, $12^{\text {th }}$ Ed. Publishers B.I. Churchil Livingstone Pvt. Ltd.sssss

3. Farquhar CM. Ectopic pregnancy. Lancet 2005;366(9485):58391.

4. Shukla DB, Jagtap SV, Kale PP, Thakkar HN. Study of ectopic pregnancy in a tertiary care centre. Int J Reprod Contracept Obstet Gynecol. 2017;6(3):975-9.
5. Asuri SS, Kalpana P. A clinical study of ectopic pregnancy. International Journal of Reproduction, Contraception, Obstet Gynecol 2016;5(11):3750-3.

6. Porwal Sanjay, Gupta R, Swarnkar M. Incidence, trends and risk factors of ectopic pregnancy in tertiary care hospital in Rajasthan. J Pharm Biomed sci 2012;16:1-3.

7. Alsuleiman SA, Grimes EM. Ectopic pregnancy: a review of 147 cases. J Reprod Med. 1982 Feb. 27(2)101-6.

8. Rashmi A, GaddagiRA, Chandrasekhar AP. A clinical study of ectopic pregnancy. J Clin Diagn Res 2012;6:867-89.

9. Barnhart KT Clinical practice. Ectopic pregnancy. $N$ Engl J Med 2009;23; 361(4):379-87.

10. S Tahmina et al. Clinical analysis of ectopic pregnancies in a tertiary care Centre in southern India: A six years retrospective study. J Clin Diagn Res 2016;10(10): QC13-QC16.

11. national health portal, ectopic pregnancy, April 2017.

How to cite this article: Gupta $\mathrm{P}$, Wadhwa $\mathrm{SN}$, Clinical course of ectopic pregnancy in a tertiary center: Observational study. Indian $J$ Obstet Gynecol Res 2019;6(2):208-210. 